\title{
Agar Gel Alkaline Electrophoresis As A Cost Effective Screening Modality For Abnormal Hemoglobins
}

\author{
Babu Rao Rentapalli ${ }^{1}$, Jayalakshmi Gade ${ }^{2}$, Md Dawood Sulemani ${ }^{3}$, \\ Suresh Babu Ganji ${ }^{4 *}$, Pavani Kiranmai Thandu ${ }^{5}$, Suneetha Revupalli ${ }^{6}$, \\ Sudhakar Kavali ${ }^{7}$, Anitha Ramtenki ${ }^{8}$ \\ ${ }^{1}$ Associate Professor of Biochemistry, ${ }^{2}$ Biochemist, ${ }^{3}$ Professor of Biochemistry, Gandhi medical college; \\ ${ }^{4}$. Assistant professor, *corresponding author email:sushwasa@ gmail.com \\ ${ }^{5}$ Senior Resident ; ${ }^{6,7}$ Biochemist $;{ }^{8}$ Post Graduate; , Department Of Biochemistry, Niloufer Hospital, Osmania \\ Medical College, Hyderabad, Telangana, India.
}

\begin{abstract}
Alkaline agar gel electrophoresis is a basic manual laborious technique for identifying Hemoglobin variants in the screening for Hemoglobinopathies. It's a cheaper alternative to the now preferred method of detection of $\mathrm{Hb}$ variants, i.e., HPLC.

In this study, at a government hospital clinical biochemistry laboratory setup, we try to compare and emphasize the importance of this manual procedure. We have done this observation from the archives of lab and case sheet data for the past four and half years; that 1891 electrophoresis were done and 65\% were normal, $20 \%$ shown Thalassemia trait, and $6 \%$ were Thalassemia major and $8 \%$ were shown sickle Hb pattern. Majority correlated clinically with the electrophoresis report and in doubtful cases (1.32\%), HPLC confirmed the electrophoresis report.

Hence wherever feasible, we recommend this manual alkaline agar gel electrophoresis where cost is a limiting factor.

Keywords: Agar gel alkaline electrophoresis; HPLC, High Performance Liquid Chromatography; SCD, Sickle cell disease; Thalassemia.
\end{abstract}

\section{Introduction}

Abnormalities of hemoglobin $(\mathrm{Hb})$ synthesis are among the most common inherited disorders of either quantitative (thalassemia syndrome) or qualitative (variant $\mathrm{Hbs}$ ) (1). The laboratory diagnosis of hemoglobinopathies and thalassemias is of growing importance, particularly because of an increasing requirement for antenatal diagnosis of significant disorders of globin chain synthesis (2). The identity of a hemoglobin variant is generally inferred from its electrophoretic mobility, its quantity, and the patient's ethnic background (3-5). Alkaline and acid hemoglobin electrophoresis is the most widely used methods for investigating hemoglobin variants and hemoglobinopathy. Alkaline electrophoresis is a reproducible method which is capable of separating common hemoglobin variants, such as hemoglobin $\mathrm{A}(\mathrm{Hb} \mathrm{A}), \mathrm{Hb} \mathrm{F}, \mathrm{Hb} \mathrm{S}$ and $\mathrm{Hb} \mathrm{C}$ but $\mathrm{Hb} \mathrm{S}, \mathrm{Hb}$ D, $\mathrm{Hb} \mathrm{G}$ and $\mathrm{Hb}$ Lepore are unresolved from each other, as are $\mathrm{Hb} \mathrm{C}, \mathrm{Hb} \mathrm{A}, \mathrm{Hb} \mathrm{O}-\mathrm{Arab}$, and $\mathrm{Hb}$ E. In addition, there are other variants with electrophoretic motilities identical or similar to those of $\mathrm{Hb}$ $\mathrm{S}$ and $\mathrm{Hb} \mathrm{C}$. Consequently, acid electrophoresis is needed for the identification of the aforementioned variants (4). Hemoglobin fraction analysis by cation-exchange HPLC has become the preferred method for quantifying $\mathrm{Hb} \mathrm{F}$ and $\mathrm{Hb} \mathrm{A} 2$ along with hemoglobin variant screening as a routine in the recent years.

In our hospital, Agar gel alkaline electrophoresis is the technique still in use for detection of thalassemias and hemoglobin variants, since long time. All the doubtful results of this screening were subjected to HPLC for further confirmation as a routine practice. Hence our study is designed to compare the results of electrophoresis with clinical condition and or reports of HPLC in diagnosing hemoglobin disorders.

\section{Materials \& Methods}

This is an observational study from the archives of laboratory records and case sheets at Niloufer hospital for women\& children, a Government hospital. A total of 1902 patients samples were received for hemoglobin electrophoresis at Clinical Biochemistry Laboratory, from January 2012 to august 2016 (duration of four and half years), were included in this study. Out of those 1902 samples, 6 were severely hemolysed, one has severe iron deficiency anemia and 4 samples were insufficient for the analysis hence those 11 samples were discarded. Hb electrophoresis was done for the remaining 1891 samples and electrophoretic patterns observed for abnormalities. These samples mainly constitute of pediatric age group or parents of those children in which carrier testing has to be done. Full case history including family history, ethnicity was noted. Complete blood picture, Sickling test results were verified from case records wherever necessary, in aiding the diagnosis. 
Electrophoresis was performed by GOLDBERG method, on Biotech ${ }^{\circledR}$ electrophoretic chamber with manually prepared agarose gel slide (6). For all the samples, hemosylate was prepared and HbF\% calculated by JONXIS method (7). Electrophoresis was performed for all the samples and patterns observed. Those samples whose diagnosis were 'doubtful' or need further identification like Hb E trait or Sickle $\beta$ Thalassemia were only sent for analysis on automated HPLC (VARIANT TM, Bio-Rad) system (with the Beta-thalassemia Short Program was used) at outside resources and the reports were obtained by the clinical staff and were filed in the patient case records.

\section{Results}

Distribution of the samples was shown in figure-1, along with their diagnosis, and the number of cases was given in the table-1.

Fig- 1.Distribution of cases as per the diagnosis

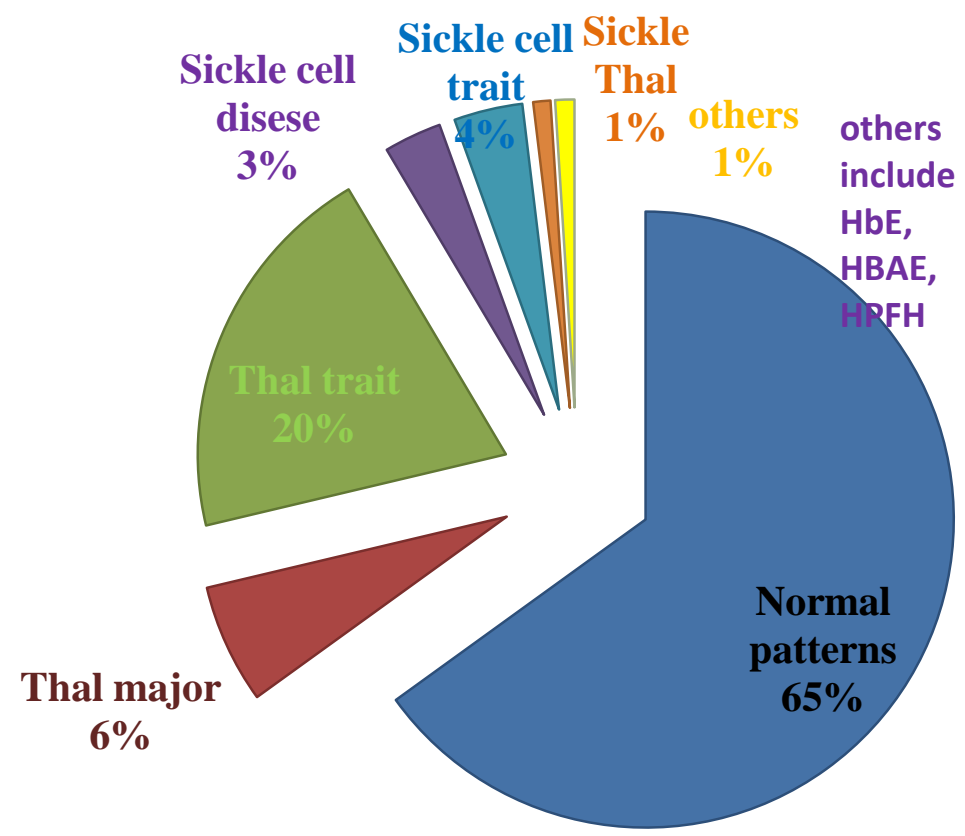

TOTAL CASES 1891

Table-1a. Distribution of cases by Diagnosis

\begin{tabular}{|c|c|c|}
\hline S. NO & DIAGNOSIS & No. of cases \\
\hline 1 & Normal patterns & 1229 \\
\hline 2 & Thalassemia major & 118 \\
\hline 3 & Thalassemia trait & 381 \\
\hline 4 & Sickle cell disease & 59 \\
\hline 5 & Sickle cell trait & 68 \\
\hline 6 & Sickle B Thalassemia & 17 \\
\hline 7 & Hb E disease & 8 \\
\hline 8 & Hb AE & 5 \\
\hline 9 & HPFH & $\mathbf{1 8 9 1}$ \\
\hline
\end{tabular}

Table-1b. Distribution of Thalassemia

\begin{tabular}{|c|c|c|c|}
\hline \multirow{2}{*}{} & \multirow{2}{*}{$\begin{array}{c}\text { Thalassemia } \\
\text { major }\end{array}$} & \multicolumn{2}{|c|}{ Thalassemia trait } \\
\cline { 3 - 4 } & \multirow{2}{*}{$\begin{array}{c}\text { Nediatric } \\
\text { patients }\end{array}$} & $\begin{array}{c}\text { parents of } \\
\text { Thalassemia } \\
\text { major patients }\end{array}$ \\
\hline cases & 118 & 61 & 320 \\
\cline { 3 - 4 } & \multicolumn{2}{|c|}{ total 381} \\
\hline
\end{tabular}


Out of the 1891 samples run, 1229 samples were shown normal. Thalassemia major and trait were 118 and 381 respectively. Out of 118 Thalassemia major cases, 6 were known cases that are already on treatment and remaining all were correlating clinically with the clinical diagnosis. Out of 381 thalassemia traits (high A2 and $\mathrm{Hb}$ A present), 61 were from pediatric patients and 320 were from the parents of thalassemia major cases. Only one pediatric patient sample has to be differentiated from HbE trait and further analysis by HPLC was negative for $\mathrm{Hb} \mathrm{E}$ trait (i.e., $\mathrm{Hb} \mathrm{AE}$ ). There were 13 cases with high density A2 zone bands with a high index of suspicion were sent for further analysis were confirmed the presence of $\mathrm{Hb} \mathrm{E}$ and were diagnosed as HbEE: 8 cases and HbAE: 5 cases.

The 6 cases with increased $(>20 \%) \mathrm{HbF}$ and with $\mathrm{Hb}$ A band and normal A2 were given a diagnosis of syndrome of Hereditary Persistence of Fetal Hemoglobin, i.e., HPFH and were well correlated with clinical features and mild degree of anemia, were again confirmed with HPLC. Sickle cell disease (SCD) and trait (SCT) were found to be 59 and 68 respectively. All SCD samples were correlating with clinical and pathological diagnosis. Out of 68 SCTs, one has to be differentiated from Sickle beta Thalassemia with doubtful $\mathrm{Hb} \mathrm{A}_{2}$ and A concentrations, needed confirmation by HPLC. There were 17 cases of Sickle beta Thalassemia in which 4 were doubtful and further needed confirmation by HPLC. These variants are shown in Fig $2 \mathrm{a} \& 2 \mathrm{~b}$.

Fig-2a. Agar gel Hemoglobin electrophoresis in alkaline pH. Hb variants are shown.

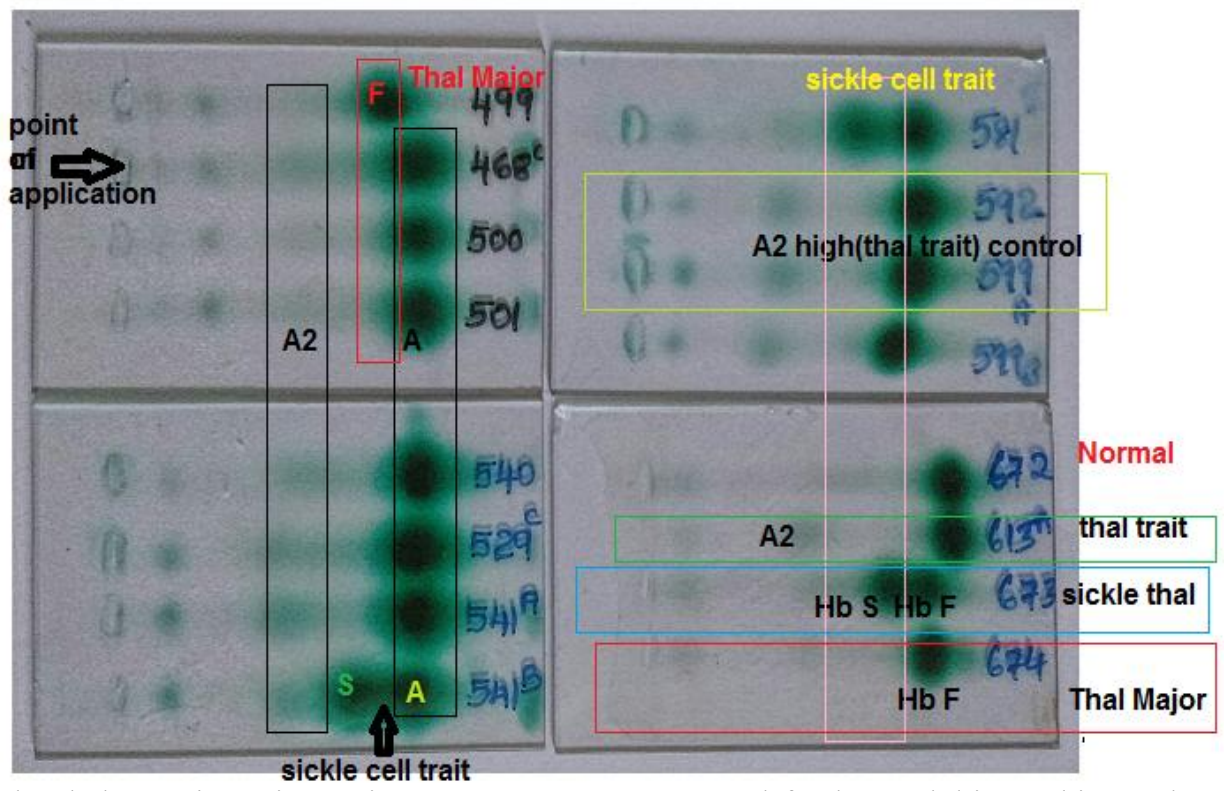

All the thalassemia major patients' parents were screened for hemoglobinopathies and were found to have thalassemia trait by alkaline gel electrophoresis. These samples were not sent for further confirmation by the HPLC analysis as they were found to have only a mild degree of anemia. All the 25 cases (1.32\%) sent for HPLC out of 1891 were only further confirming the electrophoresis pattern and none differed from what was expected.

Fig 2b. Hemoglobin electrophoresis showing $\mathrm{Hb} \mathrm{E}$ and Sickle cell homozygous pattern.

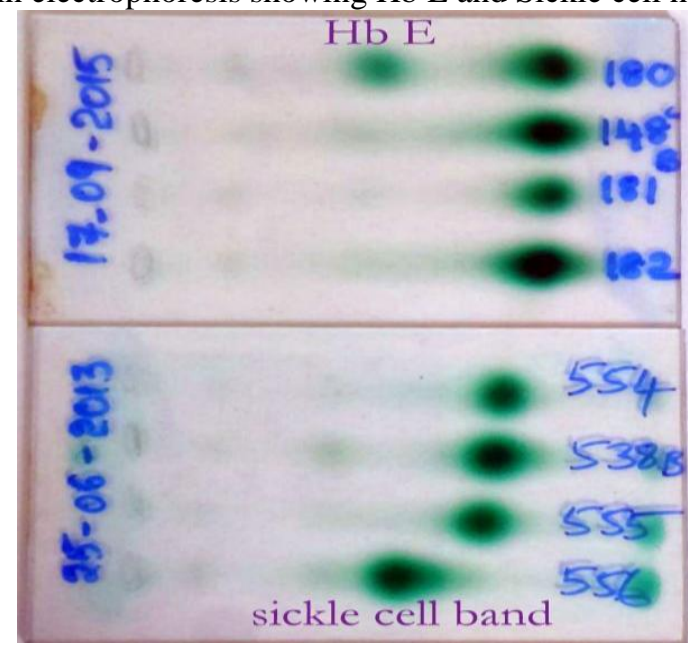




\section{Discussion}

Hemoglobin abnormalities are the most frequent genetic disease, affecting approximately $7 \%$ of the world population. Abnormal $\mathrm{Hbs}$ are generally discovered during a systematic study performed within programmes for prevention of thalassemias or sickle cell disease. In several regions (India, Turkey, Iraq, Iran, Gaza strip, Saudi Arabia, Cyprus, etc.), these are found during a premarital screening. The investigation of a patient presenting with a hematological disorder such as a chronic hemolytic anemia, an unexplained polycythaemia or a permanent cyanosis is another, completely different condition, leading to the discovery of an abnormal $\mathrm{Hb}(8,9)$. Most of the samples received in our laboratory were mainly of this type rather than for premarital screening. Electrophoresis, a test based on the migration of electrically charged molecules under an applied electric field, occupies one of the most important places in the history of abnormal $\mathrm{Hb}$ detection (9). The identification of hemoglobin variants is often presumptive, based on characteristic electrophoretic mobility, quantity, and/or ethnic origin. Fig $2 \mathrm{a}, 2 \mathrm{~b} \& 3$ shows various electrophoretic patterns and the apparatus along with our staff, respectively.

In our study we tried to compare the results obtained by basic gel electrophoretic technique with the gold standard HPLC method, which had replaced electrophoretic techniques in most labs of the India. Our results by Alkaline gel electrophoresis and HPLC technique which were similar to a study conducted by the Monica et al from Canada (19); with the suggestion that, both the techniques must be repeated after correcting iron deficiency anemia. We found no differences between alkaline gel electrophoresis and HPLC for screening hemoglobinopathies. Only $1.32 \%$ of all the cases required a further clarification by HPLC. From this, we can suggest that, in a developing country like India especially in remote places where HPLC cannot be affordable, alkaline gel electrophoresis still holds its place in screening hemoglobinopathies, with commitment for a laborious procedure.

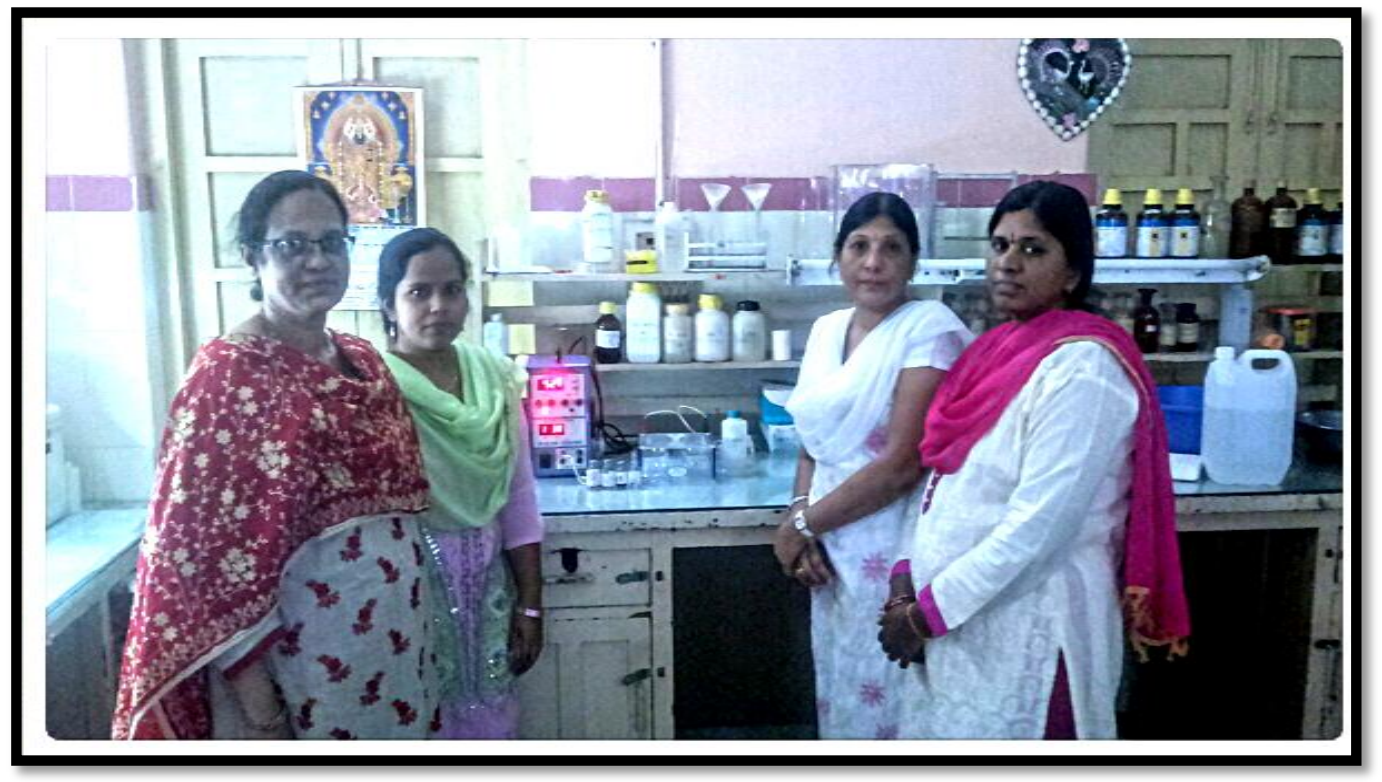

Fig 3. Hb Electrophoresis apparatus and reagents (and due respects to the committed technical staff Mrs.Sameena, Ms.Suneetha, and Mrs.Ajitha \& Mrs.Hemalatha).

Definite identification usually requires DNA analysis or amino acid sequencing (2). HPLC has been shown to be a sensitive, specific, and reproducible alternative to electrophoresis. Its use has been dramatically expanded, especially with the development of rapid, well-resolving, and fully automated analyzers. In the past decade HPLC, with its automation and its quantitative power, has appeared to be an appropriate candidate for direct identification and sensitive quantification of major and minor, normal and abnormal, hemoglobin fractions $(10,11,12-18)$. Though Alkaline electrophoresis ( $\mathrm{pH}$ 9.2) is the most common initial screening method for the detection and preliminary identification of hemoglobinopathies, Isoelectric focusing electrophoresis (IEF) has greater resolving power than conventional electrophoresis, but it is more expensive, time consuming; hence not adopted in many laboratories. HPLC technique is precise for the quantification of both $\mathrm{Hb} \mathrm{F}$ and $\mathrm{Hb} \mathrm{A}$, which made HPLC the preferred method for hemoglobinopathy and thalassemia screening for many laboratories. Capillary electrophoresis is more recent method and has advantage of quantification of $\mathrm{Hb} \mathrm{H}$, and identification of $\mathrm{Hb}$ Lepore. Electrospray mass spectrometry is becoming the method of choice for the complete characterization of newly discovered $\mathrm{Hb}$ variants. DNA analysis is used in 
the investigation of thalassemias and hemoglobinopathies to identify, in populations with a known high incidence of disease (20).

\section{Limitations of the study}

Electrophoresis in Acidic medium was not practiced in our lab, for; HPLC was the next investigation of choice.

\section{Conclusion}

Advances in technology like DNA sequencing, capillary electrophoresis and HPLC; has made the diagnosis of hemoglobinopathy easily detectable and aided in $100 \%$ confirmation of disease though they will not change the treatment options. In our study we attempted to compare the basic electrophoretic technique with HPLC and found $100 \%$ agreement between two for screening of hemoglobinopathy. Many laboratories are now using HPLC or capillary methods for screening hemoglobinopathies, but alkaline electrophoresis still holds good for the same especially in government sector or rural areas where cost is the major issue.

\section{Acknowledgements}

Authors thank Dr N.Vani, Professor and HOD of Dept.of Biochemistry, Osmania Medical College and Dr K. Ramesh Reddy, Superintendent of Niloufer Hospital.

Special thanks to our 'Guruji' Mr.Somayajulu Gollapudi garu for establishing the Hb electrophoresis technique long ago and training the staff, and we,authors dedicate this work to him with due respects.

\section{Conflict of Interest: None to declare.}

\section{References}

[1]. Kutlar F. Diagnostic approach to hemoglobinopathies. Hemoglobin. 2007;31(2):243-50

[2]. Alla Joutovsky, Joan Hadzi-Nesic, and Michael A. Nardi . HPLC Retention Time as a Diagnostic Tool for Hemoglobin Variants and Hemoglobinopathies: A Study of 60000 Samples in a Clinical Diagnostic Laboratory. Clinical Chemistry 2004; 50:10: 17361747

[3]. Working Party of the General Haematology Task Force of the British Committee for Standards in Haemotology. Guideline: the laboratory diagnosis of haemoglobinopathies. Br J Haematol 1998;101:783-92.

[4]. Bain BJ. Hemoglobinopathy diagnosis. Oxford, England: Blackwell Science Ltd., 2001:260pp.

[5]. Clarke G, Higgins TN. Laboratory investigation of hemoglobinopathies and thalassemia: review and update. Clin Chem 2000;46:1284-90.

[6]. C. A. J. Goldberg; Identification of Human Hemoglobins; Clinical Chemistry, 1957, 3 (1) 1-19.

[7]. H. P. JONXIS, T. H. J. HUISMAN; The Detection and Estimation of Fetal Hemoglobin by Means of the Alkali Denaturation, Test J Blood Nov 1956, 11 (11) 1009-1018.

[8]. Weatherall DJ, Clegg JB. Inherited haemoglobin disorders: an increasing global health problem. Bull World Health Organ. 2001;79:704-12

[9]. Henri Wajcman \& Kamran Moradkhani, Abnormal haemoglobins: detection \& characterization. Indian J Med 2011;134:538-546

[10]. Ou C-N, Rognerud CL. Diagnosis of hemoglobinopathies: electrophoresis vs. HPLC. Clin Chim Acta 2001;313:187-94.

[11]. Riou J, Godart C, Didier H, Mathis M, Bimet C, Bardakdjian-Michau J, et al. Cation-exchange HPLC evaluated for presumptive identificationof hemoglobin variants. Clin Chem 1997;43:34-9.

[12]. van der Dijs FPL, van den Berg GA, Schermer JG, Muskiet FD, Landman H, Muskiet FAJ. Screening cord blood for hemoglobinopathies and thalassemia by HPLC. Clin Chem 1992;38:1864-9.

[13]. Eastman JW, Wong R, Liao CL, Morales DR. Automated HPLC screening of newborns for sickle cell anemia and other hemoglobinopathies. Clin Chem 1996;42:704-10.

[14]. Campbell M, Henthron JS, Davies SC. Evaluation of cationexchange HPLC compared with isoelectric focusing for neonatal hemoglobinopathy screening. Clin Chem 1999;45:969-75.

[15]. Eastman JW, Lorey F, Arnopp J, Currier RJ, Sherwin J, Cunningham G. Distribution of hemoglobin F, A, S, C, E and D quantities in 4 million newborn screening specimens. Clin Chem 1999;45:683-5.

[16]. Mario N, Baudin B, Aussel C, Giboudeau J. Capillary isoelectric focusing and high-performance cation-exchange chromatography compared for qualitative and quantitative analysis of hemoglobin variants. Clin Chem 1997;43:2137-42.

[17]. Cotton F, Lin C, Fontaine B, Gulbis B, Janssens J, Vertongen F. Evaluation of a capillary electrophoresis method for routine determination of hemoglobins A2 and F. Clin Chem 1999;45:237-43.

[18]. Fucharoen S, Winichagoon P, Wisedpanichkij R, Sae-Ngow B, Sriphanich R, Oncoung W, et al. Prenatal and postnatal diagnoses of thalassemias and hemoglobinopathies by HPLC. Clin Chem1998;44:740-8.

[19]. Monica Dogaru, Daniel Coriu1, Trefor Higgins Comparison of two analytical methods (electrophoresis and HPLC) to detect thalassemias and hemoglobinopathies Revista Română de Medicină de Laborator., 2007;9: 39-47.

[20]. Tietz textbook of clinical chemistry and molecular diagnostics, chapter 32; pages 1003-1006. 